\title{
AKARI Infrared Views of AGNs
}

\author{
Shinki Oyabu ${ }^{1}$ \\ ${ }^{1}$ Institute of Space and Astronautical Science, Japan Aerospace Exploration Agency, \\ 3-1-1 Yosinodai, Sagamihara, Kanagawa 229-8510, Japan \\ Email: oyabu@ir.isas.jaxa.jp
}

\begin{abstract}
The Japanese infrared satellite $A K A R I$ has unique capabilities for near-infrared spectroscopy and an all-sky survey in the mid- and far-infrared. We present the recent results on active galactic nuclei that use the unique capabilities of $A K A R I$.
\end{abstract}

Keywords. galaxies: active, infrared: galaxies, quasars: emission lines

\section{AKARI, the Japanese Infrared Satellite}

$A K A R I$, the Japanese infrared satellite, launched in 2006 February, has a 70-cm primary mirror cooled with the combination of liquid helium and mechanical coolers. It has unique capabilities for near-infrared spectroscopy in the wavelength range $2 \mu \mathrm{m}$ to $5 \mu \mathrm{m}$, and carried out an all-sky survey in the mid- and far-infrared. It has broadband filters comprehensively covering near-, mid-, and far-infrared wavelengths (Murakami et al. 2007).

\section{Near-Infrared Spectroscopy of High-Redshift Quasars}

Near-infrared spectroscopy of the high-redshift quasars RX J1759.4+6638 at $z=4.3$ and APM $08279+5255$ at $z=3.9$ led to detection of the $\mathrm{H} \alpha$ emission line at the highest redshift to date (Oyabu et al. 2007; Oyabu et al. 2009). In addition, for APM 08279+5255, the hydrogen recombination emission lines $\mathrm{Pa} \alpha$ and $\mathrm{Pa} \beta$ as well as the optically thick blackbody emission which comes from the inner part of dust torus were detected. Neither quasar provided any suggestion of evolution when compared with low-redshift quasars.

\section{Mid-Infrared Search for Active Galactic Nuclei}

Using the point source catalog from the $A K A R I$ mid-infrared all-sky survey, we are searching for AGNs, not only for normal AGNs but also for dusty AGNs, in the local Universe. Our detection limits in the mid-infrared all-sky survey reach $50 \mathrm{mJy}$ and $120 \mathrm{mJy}$ in $9 \mu \mathrm{m}$ and $18 \mu \mathrm{m}$ bands, respectively (Ishihara et al. 2009). AKARI provides remarkable improvement in sensitivity and spatial resolution compared to the previous all-sky survey with IRAS. Red mid-infrared sources away from the Galactic plane are observed using the near-infrared spectroscopy capability that remains since the cyrogenic helium was exhausted. During these follow-up observations, we have started to detect hidden AGNs located in galaxies in which AGN activity was not recognized at other wavelengths (Oyabu et al. in preparation).

\section{References}

Ishihara, D., et al. 2009, Astronomical Society of the Pacific Conference Series, 418, 9

Murakami, H., et al. 2007, PASJ, 59, 369

Oyabu, S., et al. 2007, PASJ, 59, 497

Oyabu, S., Kawara, K., Tsuzuki, Y., Matsuoka, Y., Sameshima, H., Asami, N., \& Ohyama, Y. 2009, ApJ, 697, 452 